RESEARCH ARTICLE

\title{
Management of sucking pests in rabi/summer groundnut using newer molecule insecticides
}

\author{
Indiragandhi Pandiyan* \\ *Horticulture College and Research Institute, Tamil Nadu Agricultural University, Periyakulam
}

\begin{abstract}
The field experiments were conducted at Regional Research Station, Vriddhachalam, during rabi/summer season for three consecutive years from 2012 to 2015 to test the efficacy of newer molecules insecticides against sucking insect pests thrips and leafhopper in groundnut. Among the seven newer molecule insecticides tested, imidacloprid 200SL @ 200 $\mathrm{ml} \mathrm{ha}^{-1}$ was found to be effective in reducing thrips damage (16\%) followed by thiamethoxam 25WG @ 200gm ha-1 (18\%) as against 33\% in control. Imidacloprid 200SL and acetamiprid 20\%SP@100 gm ha ${ }^{-1}$ registered less incidence of leafhopper (15\%) compared to control (32\%). Therefore, acetamiprid 20\%SP @ 100 gm ha ${ }^{-1}$, thiamethoxam 25WG @100 gm.ha-1 gm/ha and imidacloprid 200SL @ $200 \mathrm{ml}^{\text {. ha }}{ }^{-1}$ were found to be effective against thrips, leafhopper population and its damage. These three molecules realized more dry pod $\left(2274 \mathrm{~kg} / \mathrm{ha}^{-1}, 2013 \mathrm{~kg} \mathrm{ha}^{-1}, 2100 \mathrm{~kg} \mathrm{ha}^{-1}\right)$ and haulm yield $\left(8.2 \mathrm{t} \mathrm{ha}^{-1}, 7.6 \mathrm{t} \mathrm{ha}^{-1}, 7.8 \mathrm{t} \mathrm{ha}^{-1}\right)$ and ultimately the benefit cost ratio of $1: 2.8 ; 1: 2.4$ and $1: 2.5$ respectively. However, imidacloprid $200 \mathrm{SL}$ and thiamethoxam 25WG $200 \mathrm{ml} \mathrm{ha}^{-1}$ recorded more number of predatory coccinellids ( 0.51 and 0.40 plant $\left.^{-1}\right)$, spider $\left(0.27\right.$ and 0.24 plant $\left.^{-1}\right)$, spider egg mass ( 0.10 and 0.12 plant $\left.^{-1}\right)$. Thus, the use of imdiacloprid 200SL or thiamethoxam 25WG individually or incorporation of these chemicals in an integrated pest management programme for sucking pests on groundnut may prove as economically viable with less effect on natural enemies in groundnut eco-system during rabi/summer seasons.
\end{abstract}

Received : $10^{\text {th }}$ August, 2020

Revised : $24^{\text {th }}$ August, 2020

Accepted : $08^{\text {th }}$ September, 2020

Keywords: acetamiprid; imidacloprid; thiamethoxam; coccinellids; spiders; groundnut.

\section{INTRODUCTION}

Groundnut (Arachis hypogaea L.) is one of the major oilseed crops cultivated in about eight million hectares, with an annual production of over nine million tonnes of pods, contributing $45 \%$ of oilseed production in India. In India, which is mainly grown in the southern and north-western states, Gujrat, Andhra Pradesh, Tamil Nadu, Karnataka, Maharastra, and Madhya Pradesh, together occupying about 90 percent of the groundnut area in the country. Insect pest menace is one of the few essential biotic stresses contributing towards lower yield. Sucking pests are the major biotic constraints in groundnut production. The major sucking insect pests of groundnut comprise of thrips (Frankliniella hultzeri Trybom, Thrips palmi Karny and Scirtothrips dorsalis Hood), leafhopper (Empoasca kerri Pruthi) (David and Ramamurthy, 2011). Thrips are the important sucking pests that live in the flowers and folded leaflets of groundnut known to cause yield loss and also responsible for spreading bud necrosis, a viral disease in groundnut. Leafhoppers suck the sap from the leaves and petioles and mainly prefer the first three-terminal leaves and feeding symptoms induce the yellowing of foliage that begins at the tip, known as hopper burn (Khan and Hussain, 1965). A heavy infestation of sucking pests on young plants results in considerable damage both by direct injury and by the transmission of diseases such as bud necrosis and rosette. Thrips and jassids are considered as important destructive pests on this crop during rabi/summer season. Keeping this in view, a study was undertaken to test the effectiveness of some newer molecule insecticides against these pests in groundnut.

\section{MATERIAL AND METHODS}

The field experiments were carried out during rabi/summer seasons of 2012-13, 2013-14, 201415 at Regional Research Station, Vriddhachalam (11 $\left.30^{\prime} 0.00^{\prime} \mathrm{N} ; 79^{\circ} 19^{\prime} 48.00^{\prime \prime} \mathrm{E}\right)$ using the popular groundnut variety VRI 2 . The crop was sown at the spacing of $30 \mathrm{~cm} \times 10 \mathrm{~cm}$. All the recommended package of practices was followed 
except the plant protection measures. Treatments are acephate $75 \%$ SP @ $1.30 \mathrm{~kg} \mathrm{ha}^{-1}$, acetamiprid 20\%SP $100 \mathrm{gm} \mathrm{ha}^{-1}$, fipronyl 5\%SC $100 \mathrm{gm} \mathrm{ha}^{-1}$, imidacloprid 200SL @ $200 \mathrm{ml} \mathrm{ha}^{-1}$, thiamethoxam 25WG @ $200 \mathrm{gm} \mathrm{ha}^{-1}$, thiochloprid 480SC @ 200 ml ha-1, triazophos 25EC @ 2 lit ha-1, and control. The treatments were tested in a randomized Block Design with three replications. The treatments were imposed whenever the population of thrips and leafhopper appeared or on 30 day after sowing (DAS), whichever is earlier. Spraying was applied with the help of a manually operated knapsack sprayer. Thrips and leafhopper population was recorded before 24 hours and 7 and 15 days after spraying (DAS). Observations on the number of thrips $/ 3$ terminal leaves/plant and a number of hoppers/ leaves/plant were made on top, middle and bottom leaves of 10 randomly selected plants from each replication. Natural enemies like coccinellids, spiders population and its egg mass were also recorded. While harvesting, pod and haulm yield were recorded to work out the cost-benefit ratio. Data were statistically analyzed using OPSTAT (Sheoran et al., 1998).

\section{RESULTS AND DISCUSSION}

The reduction of sucking pests such as thrips and leafhopper population and their damage percent by the different newer molecule insecticides imposed on groundnut is given in Tables 1 and 2 .

Table 1.Effect of newer molecules against thrips in groundnut during rabi/Summer seasons (2012-2015)

\begin{tabular}{|c|c|c|c|c|c|c|c|c|c|c|c|c|c|c|c|c|c|c|c|}
\hline \multirow{3}{*}{ Treatment } & \multirow{3}{*}{ Doseha $^{1}$} & \multicolumn{9}{|c|}{ No. of thrips $/ 3$ terminal leaves $/$ plant } & \multicolumn{9}{|c|}{ Thrips damage (\%) } \\
\hline & & \multicolumn{3}{|c|}{$2012-2013$} & \multicolumn{3}{|c|}{ 2013-2014 } & \multicolumn{3}{|c|}{$2014-2015$} & \multicolumn{3}{|c|}{$2012-2013$} & \multicolumn{3}{|c|}{ 2013-2014 } & \multicolumn{3}{|c|}{$2014-2015$} \\
\hline & & PTC & 7DAS & 15DAS & PTC & 7DAS & 15DAS & PTC & 7DAS & 15DAS & PTC & 7DAS & 15DAS & PTC & 7DAS & 15DAS & PTC & 7DAS & 15DAS \\
\hline T1-Acephate 75SP & $1.30 \mathrm{~kg}$ & $\begin{array}{c}5.7 \\
(2.4)\end{array}$ & $\begin{array}{l}4.2 \\
(2.0)\end{array}$ & $\begin{array}{l}4.3 \\
(2.1)\end{array}$ & $\begin{array}{c}3.5 \\
(2.1)\end{array}$ & $\begin{array}{c}2.2 \\
(1.7)\end{array}$ & $\begin{array}{c}2.5 \\
(1.8)\end{array}$ & $\begin{array}{c}8.2 \\
(3.03)\end{array}$ & $\begin{array}{l}4.5 \\
(2.3)\end{array}$ & $\begin{array}{l}4.5 \\
(2.3)\end{array}$ & $\begin{array}{c}30.0 \\
(35.2)\end{array}$ & $\begin{array}{l}27.0 \\
(31.3)\end{array}$ & $\begin{array}{l}29.0 \\
(32.5)\end{array}$ & $\begin{array}{l}22.0 \\
(27.3)\end{array}$ & $\begin{array}{l}12.0 \\
(20.2)\end{array}$ & $\begin{array}{c}10.0 \\
(17.8)\end{array}$ & $\begin{array}{l}18.0 \\
(25.1)\end{array}$ & $\begin{array}{l}10.0 \\
(18.4)\end{array}$ & $\begin{array}{l}11.0 \\
(19.3)\end{array}$ \\
\hline T2-Acetamiprid 20\%SP & $100 \mathrm{gm}$ & $\begin{array}{c}5.7 \\
(2.4)\end{array}$ & $\begin{array}{l}4.5 \\
(2.1)\end{array}$ & $\begin{array}{l}4.5 \\
(2.1)\end{array}$ & $\begin{array}{l}3.7 \\
(2.1)\end{array}$ & $\begin{array}{l}2.8 \\
(1.9)\end{array}$ & $\begin{array}{l}3.0 \\
(1.9)\end{array}$ & $\begin{array}{l}7.5 \\
(2.9)\end{array}$ & $\begin{array}{l}4.0 \\
(2.2)\end{array}$ & $\begin{array}{l}5.8 \\
(2.6)\end{array}$ & $\begin{array}{l}30.0 \\
(35.2)\end{array}$ & $\begin{array}{l}28.0 \\
(31.9)\end{array}$ & $\begin{array}{l}29.0 \\
(32.5)\end{array}$ & $\begin{array}{l}22.0 \\
(27.9)\end{array}$ & $\begin{array}{l}15.0 \\
(22.7)\end{array}$ & $\begin{array}{l}13.0 \\
(21.1)\end{array}$ & $\begin{array}{l}15.0 \\
(22.7)\end{array}$ & $\begin{array}{l}12.0 \\
(20.2)\end{array}$ & $\begin{array}{l}12.0 \\
(20.2)\end{array}$ \\
\hline T3-Fipronyl5\% SC & $100 \mathrm{gm}$ & $\begin{array}{l}5.7 \\
(2.4)\end{array}$ & $\begin{array}{l}4.5 \\
(2.1)\end{array}$ & $\begin{array}{l}4.6 \\
(2.1)\end{array}$ & $\begin{array}{l}4.8 \\
(2.4)\end{array}$ & $\begin{array}{l}3.2 \\
(2.0)\end{array}$ & $\begin{array}{l}3.5 \\
(2.0)\end{array}$ & $\begin{array}{l}8.0 \\
(3.0)\end{array}$ & $\begin{array}{l}5.2 \\
(2.5)\end{array}$ & $\begin{array}{l}6.5 \\
(2.7)\end{array}$ & $\begin{array}{l}32.0 \\
(35.9)\end{array}$ & $\begin{array}{l}30.01 \\
(33.2)\end{array}$ & $\begin{array}{c}31.0 \\
(33.8)\end{array}$ & $\begin{array}{l}26.0 \\
(30.6)\end{array}$ & $\begin{array}{l}18.0 \\
(25.0)\end{array}$ & $\begin{array}{l}14.0 \\
(21.9)\end{array}$ & $\begin{array}{l}18.0 \\
(25.1)\end{array}$ & $\begin{array}{l}16.0 \\
(23.5)\end{array}$ & $\begin{array}{l}18.0 \\
(25.1)\end{array}$ \\
\hline T4-Imidacloprid 200SL & $200 \mathrm{ml}$ & $\begin{array}{c}5.7 \\
(2.4)\end{array}$ & $\begin{array}{l}4.3 \\
(2.1)\end{array}$ & $\begin{array}{l}4.3 \\
(2.1)\end{array}$ & $\begin{array}{l}4.6 \\
(2.4)\end{array}$ & $\begin{array}{c}2.5 \\
(1.8)\end{array}$ & $\begin{array}{l}3.2 \\
(2.0)\end{array}$ & $\begin{array}{l}7.5 \\
(2.8)\end{array}$ & $\begin{array}{l}3.2 \\
(2.0)\end{array}$ & $\begin{array}{l}3.8 \\
(2.2)\end{array}$ & $\begin{array}{l}34.0 \\
(36.6)\end{array}$ & $\begin{array}{l}27.0 \\
(31.3)\end{array}$ & $\begin{array}{l}29.0 \\
(32.5)\end{array}$ & $\begin{array}{l}22.0 \\
(27.9)\end{array}$ & $\begin{array}{l}12.0 \\
(20.2)\end{array}$ & $\begin{array}{l}12.0 \\
(20.2)\end{array}$ & $\begin{array}{l}15.0 \\
(22.7)\end{array}$ & $\begin{array}{c}8.0 \\
(16.4)\end{array}$ & $\begin{array}{c}8.0 \\
(16.4)\end{array}$ \\
\hline $\begin{array}{l}\text { T5-Thiamethoxam } \\
\text { 25WG }\end{array}$ & $200 \mathrm{gm}$ & $\begin{array}{c}5.7 \\
(2.4)\end{array}$ & $\begin{array}{l}4.3 \\
(2.1)\end{array}$ & $\begin{array}{l}4.4 \\
(2.1)\end{array}$ & $\begin{array}{l}5.2 \\
(2.4)\end{array}$ & $\begin{array}{c}3.0 \\
(1.9)\end{array}$ & $\begin{array}{c}3.0 \\
(1.9)\end{array}$ & $\begin{array}{l}8.0 \\
(3.0)\end{array}$ & $\begin{array}{c}3.8 \\
(2.2)\end{array}$ & $\begin{array}{l}4.2 \\
(2.2)\end{array}$ & $\begin{array}{c}34.0 \\
(36.6)\end{array}$ & $\begin{array}{l}31.0 \\
(33.8)\end{array}$ & $\begin{array}{l}30.0 \\
(33.2)\end{array}$ & $\begin{array}{l}20.0 \\
(26.5)\end{array}$ & $\begin{array}{l}12.0 \\
(20.2)\end{array}$ & $\begin{array}{l}12.0 \\
(20.2)\end{array}$ & $\begin{array}{l}16.0 \\
(23.5)\end{array}$ & $\begin{array}{l}12.0 \\
(20.2)\end{array}$ & $\begin{array}{l}13.0 \\
(21.1)\end{array}$ \\
\hline T6-Thiochloprid 480SC & $200 \mathrm{ml}$ & $\begin{array}{l}5.6 \\
(2.4)\end{array}$ & $\begin{array}{l}4.5 \\
(2.1)\end{array}$ & $\begin{array}{l}4.5 \\
(2.1)\end{array}$ & $\begin{array}{l}5.0 \\
(2.4)\end{array}$ & $\begin{array}{l}3.2 \\
(2.0)\end{array}$ & $\begin{array}{l}3.2 \\
(2.0)\end{array}$ & $\begin{array}{c}8.2 \\
(3.0)\end{array}$ & $\begin{array}{l}4.0 \\
(2.2)\end{array}$ & $\begin{array}{l}5.2 \\
(2.4)\end{array}$ & $\begin{array}{c}32.0 \\
(35.9)\end{array}$ & $\begin{array}{c}30.0 \\
(33.2)\end{array}$ & $\begin{array}{c}31.0 \\
(33.8)\end{array}$ & $\begin{array}{c}20.0 \\
(26.5)\end{array}$ & $\begin{array}{l}14.0 \\
(21.9)\end{array}$ & $\begin{array}{l}14.0 \\
(21.9)\end{array}$ & $\begin{array}{l}16.0 \\
(23.5)\end{array}$ & $\begin{array}{l}12.0 \\
(20.2)\end{array}$ & $\begin{array}{l}13.0 \\
(21.1)\end{array}$ \\
\hline T7-Triazophos 25EC & 2.0 lit & $\begin{array}{c}5.6 \\
(2.4)\end{array}$ & $\begin{array}{l}4.5 \\
(2.1)\end{array}$ & $\begin{array}{c}4.4 \\
(2.1)\end{array}$ & $\begin{array}{c}5.5 \\
(2.5)\end{array}$ & $\begin{array}{c}4.0 \\
(2.2)\end{array}$ & $\begin{array}{c}4.2 \\
(2.2)\end{array}$ & $\begin{array}{c}8.5 \\
(3.0)\end{array}$ & $\begin{array}{l}4.8 \\
(2.4)\end{array}$ & $\begin{array}{l}5.0 \\
(2.4)\end{array}$ & $\begin{array}{c}31.0 \\
(35.5)\end{array}$ & $\begin{array}{c}29.0 \\
(32.6)\end{array}$ & $\begin{array}{c}33.0 \\
(35.1)\end{array}$ & $\begin{array}{c}20.0 \\
(26.5)\end{array}$ & $\begin{array}{l}14.0 \\
(21.9)\end{array}$ & $\begin{array}{l}15.0 \\
(22.7)\end{array}$ & $\begin{array}{l}15.0 \\
(22.7)\end{array}$ & $\begin{array}{l}13.0 \\
(21.1)\end{array}$ & $\begin{array}{c}20.0 \\
(25.8)\end{array}$ \\
\hline T8-Control & & $\begin{array}{l}5.7 \\
(2.4)\end{array}$ & $\begin{array}{l}6.8 \\
(2.6)\end{array}$ & $\begin{array}{c}7.1 \\
(2.6)\end{array}$ & $\begin{array}{c}5.8 \\
(2.5)\end{array}$ & $\begin{array}{c}9.0 \\
(3.1)\end{array}$ & $\begin{array}{l}12.5 \\
(3.6)\end{array}$ & $\begin{array}{c}8.2 \\
(3.0)\end{array}$ & $\begin{array}{l}12.0 \\
(3.6)\end{array}$ & $\begin{array}{l}16.5 \\
(4.2)\end{array}$ & $\begin{array}{c}36.0 \\
(37.4)\end{array}$ & $\begin{array}{c}38.0 \\
(38.0)\end{array}$ & $\begin{array}{c}40.0 \\
(39.2)\end{array}$ & $\begin{array}{c}28.0 \\
(31.9)\end{array}$ & $\begin{array}{c}32.0 \\
(34.3)\end{array}$ & $\begin{array}{c}35.0 \\
(36.2)\end{array}$ & $\begin{array}{l}18.0 \\
(24.7)\end{array}$ & $\begin{array}{l}22.0 \\
(27.9)\end{array}$ & $\begin{array}{l}25.0 \\
(29.9)\end{array}$ \\
\hline CV & & 5.41 & 2.62 & 3.43 & N/A & 0.13 & 0.13 & 0.001 & 0.108 & 0.17 & 4.85 & 6.11 & 5.19 & 3.97 & 3.60 & 2.43 & N/A & 0.95 & 3.89 \\
\hline $\mathrm{SE}(\mathrm{m})$ & - & - & - & - & 0.14 & 0.04 & 0.04 & 0.001 & 0.036 & 0.05 & - & - & - & 1.33 & 1.20 & 0.81 & 0.83 & 0.32 & 1.30 \\
\hline SEd & - & 0.25 & 0.04 & 0.06 & 0.20 & 0.06 & 0.06 & 0.001 & 0.051 & 0.08 & 1.27 & 1.5 & 1.34 & 1.87 & 1.70 & 1.15 & 1.18 & 0.45 & 1.84 \\
\hline $\mathrm{CD}(0.05)$ & - & 0.52 & 0.09 & 0.12 & 10.5 & 3.68 & 3.56 & 0.03 & 2.55 & 3.73 & 2.68 & 3.16 & 2.82 & 8.00 & 8.80 & 6.13 & 6.02 & 2.56 & 9.7 \\
\hline
\end{tabular}

Values are mean of three replication of each treatment. Values in the paranthesis are square root transformed values for population and arcsine transformed values for percent damage. PTC- Pre treatment count, DAS-Days after spraying

The reduction in sucking pest population took place after imposing different newer molecule insecticides.

\section{Thrips}

Three years data indicated that significantly lower thrips population was recorded in all the treatments than in control (Table 1). However, significant reduction in thrips population (4.2/3 terminal leaves/plant) was noticed in acephate $75 \%$ SP @ $1.3 \mathrm{~kg} \mathrm{ha}^{-1} \mathrm{a}$ and it was at par with all other newer molecules when compared to control (6.8/3 terminal leaves/plant) on $7^{\text {th }}$ DAS. The effect of the newer molecules insecticides on the thrips population was stable till $15^{\text {th }}$ DAS during rabi/summer 2012 13. A similar trend was observed for rabi/summer 2013-14, where the thrips population (2.2 and 2.5/3 terminal leaves/plant) observed in acephate treated plots on $7^{\text {th }}$ and $15^{\text {th }}$ DAS respectively, was significant than in other treatments. Whereas during rabi/summer 2014-15 imidacloprid recorded significantly less thrips population 3.2 and $3.8 / 3$ terminal leaves/plant on $7^{\text {th }}$ and $15^{\text {th }}$ DAS followed by thiomethoxam (3.8 and 4.2/3 terminal leaves/plant) and acephate (4.5 and 4.5/3 terminal leaves/plant). Zadda et al.,(2015) reported that imidacloprid was effective in reducing the thrips population in rainfed groundnut crop.

With respect to thrips damage percent, significantly less damage was recorded in acephate, acetamiprid, imidacloprid (29\%) followed by thiomehoxam (30\%) during rabi/summer 201213 (Table 1). During 2013-14 also acephate recorded significantly less damage (10\%) followed by imdacloprid and thiomethoxam (12\%). When compared to previous two years rabi/summer season over all thrips damage was less during 2014-15, yet imidacloprid recorded significantly low damage $(8 \%)$, followed by acephate (11\%), acetamiprid (12\%), thiamethoxam and thiocloprid (13\%) on $15^{\text {th }}$ DAS (Table 1$)$.

Results of the present study were in agreement with the earlier findings of Nataraja et al., 2013; 
Table 2.Effect of newer molecules against jassids in groundnut during rabi/Summer seasons (2012-2015)

\begin{tabular}{|c|c|c|c|c|c|c|c|c|c|c|c|c|c|c|c|c|c|c|c|}
\hline \multirow{3}{*}{ Treatment } & \multirow{3}{*}{$\begin{array}{c}\text { Dose } \\
\text { ha }^{-1}\end{array}$} & \multicolumn{9}{|c|}{ No. of jassids $/ 3$ terminal leaves /plant } & \multicolumn{9}{|c|}{ Leafhopper damage (\%) } \\
\hline & & \multicolumn{3}{|c|}{ 2012-2013 } & \multicolumn{3}{|c|}{ 2013-14 } & \multicolumn{3}{|c|}{ 2014-2015 } & \multicolumn{3}{|c|}{ 2012-2013 } & \multicolumn{3}{|c|}{ 2013-14 } & \multicolumn{3}{|c|}{ 2014-2015 } \\
\hline & & PTC & $\begin{array}{c}7 \\
\text { DAS }\end{array}$ & $\begin{array}{c}15 \\
\text { DAS }\end{array}$ & PTC & $\begin{array}{c}7 \\
\text { DAS }\end{array}$ & $\begin{array}{c}15 \\
\text { DAS }\end{array}$ & PTC & $\begin{array}{c}7 \\
\text { DAS }\end{array}$ & $\begin{array}{c}15 \\
\text { DAS }\end{array}$ & PTC & $\begin{array}{c}7 \\
\text { DAS }\end{array}$ & $\begin{array}{c}15 \\
\text { DAS }\end{array}$ & PTC & $\begin{array}{c}7 \\
\text { DAS }\end{array}$ & $\begin{array}{c}15 \\
\text { DAS }\end{array}$ & PTC & $\begin{array}{c}7 \\
\text { DAS }\end{array}$ & $\begin{array}{c}15 \\
\text { DAS }\end{array}$ \\
\hline $\begin{array}{l}\text { T1-Acephate } \\
75 \% \text { SP }\end{array}$ & $1.30 \mathrm{~kg}$ & $\begin{array}{c}7.0 \\
(2.6)\end{array}$ & $\begin{array}{l}5.6 \\
(2.4)\end{array}$ & $\begin{array}{l}5.6 \\
(2.4)\end{array}$ & $\begin{array}{c}6.5 \\
(2.7)\end{array}$ & $\begin{array}{c}3.5 \\
(10.4)\end{array}$ & $\begin{array}{c}3.5 \\
(10.4)\end{array}$ & $\begin{array}{c}6.5 \\
(14.6)\end{array}$ & $\begin{array}{c}3.2 \\
(9.9)\end{array}$ & $\begin{array}{c}3.5 \\
(10.4)\end{array}$ & $\begin{array}{c}28.0 \\
(31.9)\end{array}$ & $\begin{array}{c}25.0 \\
(31.3)\end{array}$ & $\begin{array}{c}25.0 \\
(30.0)\end{array}$ & $\begin{array}{c}22.0 \\
(27.3)\end{array}$ & $\begin{array}{c}16.0 \\
(23.3)\end{array}$ & $\begin{array}{c}18.0 \\
(24.9)\end{array}$ & $\begin{array}{c}15.0 \\
(22.5)\end{array}$ & $\begin{array}{c}10.0 \\
(18.3)\end{array}$ & $\begin{array}{c}10.5 \\
(18.8)\end{array}$ \\
\hline $\begin{array}{l}\text { T2-Acetamiprid } \\
20 \% \text { SP }\end{array}$ & $100 \mathrm{gm}$ & $\begin{array}{l}7.2 \\
(2.6)\end{array}$ & $\begin{array}{c}4.8 \\
(2.2)\end{array}$ & $\begin{array}{l}5.3 \\
(2.3)\end{array}$ & $\begin{array}{c}6.5 \\
(2.7)\end{array}$ & $\begin{array}{c}3.2 \\
(9.9)\end{array}$ & $\begin{array}{c}2.8 \\
(9.13)\end{array}$ & $\begin{array}{c}4.5 \\
(12.0)\end{array}$ & $\begin{array}{c}2.5 \\
(8.4)\end{array}$ & $\begin{array}{c}3.5 \\
(10.4)\end{array}$ & $\begin{array}{c}28.0 \\
(31.9)\end{array}$ & $\begin{array}{c}26.0 \\
(28.6)\end{array}$ & $\begin{array}{c}25.0 \\
(30.0)\end{array}$ & $\begin{array}{c}28.0 \\
(31.8)\end{array}$ & $\begin{array}{l}12.0 \\
(19.9)\end{array}$ & $\begin{array}{c}14.3 \\
(21.9)\end{array}$ & $\begin{array}{c}18.0 \\
(25.1)\end{array}$ & $\begin{array}{c}8.0 \\
(16.3)\end{array}$ & $\begin{array}{c}8.5 \\
(16.8)\end{array}$ \\
\hline $\begin{array}{l}\text { T3-Fipronyl } \\
5 \% \text { SC }\end{array}$ & $100 \mathrm{gm}$ & $\begin{array}{c}7.4 \\
(2.7)\end{array}$ & $\begin{array}{l}5.6 \\
(2.4)\end{array}$ & $\begin{array}{l}5.6 \\
(2.4)\end{array}$ & $\begin{array}{c}6.2 \\
(2.6)\end{array}$ & $\begin{array}{c}3.8 \\
(10.9)\end{array}$ & $\begin{array}{c}4.0 \\
(11.2)\end{array}$ & $\begin{array}{c}5.0 \\
(12.7)\end{array}$ & $\begin{array}{c}3.5 \\
(10.4)\end{array}$ & $\begin{array}{c}5.5 \\
(13.4)\end{array}$ & $\begin{array}{l}25.0 \\
(30.0)\end{array}$ & $\begin{array}{l}26.0 \\
(28.6)\end{array}$ & $\begin{array}{c}26.0 \\
(30.6)\end{array}$ & $\begin{array}{c}22.0 \\
(27.8)\end{array}$ & $\begin{array}{l}18.0 \\
(24.9)\end{array}$ & $\begin{array}{l}20.0 \\
(26.4)\end{array}$ & $\begin{array}{l}15.0 \\
(22.7)\end{array}$ & $\begin{array}{c}12.0 \\
(20.2)\end{array}$ & $\begin{array}{l}12.0 \\
(20.2)\end{array}$ \\
\hline $\begin{array}{l}\text { T4-Imidacloprid } \\
\text { 200SL }\end{array}$ & $200 \mathrm{ml}$ & $\begin{array}{l}7.0 \\
(2.6)\end{array}$ & $\begin{array}{c}4.5 \\
(2.1)\end{array}$ & $\begin{array}{l}5.4 \\
(2.3)\end{array}$ & $\begin{array}{l}6.8 \\
(2.7)\end{array}$ & $\begin{array}{c}3.2 \\
(9.9)\end{array}$ & $\begin{array}{c}2.2 \\
(7.6)\end{array}$ & $\begin{array}{c}5.0 \\
(12.7)\end{array}$ & $\begin{array}{c}2.0 \\
(6.5)\end{array}$ & $\begin{array}{l}2.5 \\
(8.4)\end{array}$ & $\begin{array}{c}28.0 \\
(31.9)\end{array}$ & $\begin{array}{c}25.0 \\
(30.0)\end{array}$ & $\begin{array}{c}24.0 \\
(29.3)\end{array}$ & $\begin{array}{c}26.0 \\
(30.5)\end{array}$ & $\begin{array}{c}13.3 \\
(21.3)\end{array}$ & $\begin{array}{l}16.0 \\
(23.3)\end{array}$ & $\begin{array}{c}18.0 \\
(25.1)\end{array}$ & $\begin{array}{c}8.0 \\
(16.3)\end{array}$ & $\begin{array}{c}8.0 \\
(16.3)\end{array}$ \\
\hline $\begin{array}{l}\text { T5- } \\
\text { Thiamethoxam } \\
\text { 25WG }\end{array}$ & $200 \mathrm{gm}$ & $\begin{array}{l}6.8 \\
(2.6)\end{array}$ & $\begin{array}{l}5.4 \\
(2.3)\end{array}$ & $\begin{array}{l}5.5 \\
(2.3)\end{array}$ & $\begin{array}{c}7.5 \\
(2.9)\end{array}$ & $\begin{array}{c}3.5 \\
(10.4)\end{array}$ & $\begin{array}{c}3.2 \\
(9.9)\end{array}$ & $\begin{array}{c}5.0 \\
(12.7)\end{array}$ & $\begin{array}{c}3.0 \\
(9.5)\end{array}$ & $\begin{array}{c}3.5 \\
(10.4)\end{array}$ & $\begin{array}{c}30.0 \\
(33.2)\end{array}$ & $\begin{array}{c}24.0 \\
(29.3)\end{array}$ & $\begin{array}{c}26.0 \\
(31.9)\end{array}$ & $\begin{array}{c}25.7 \\
(30.1)\end{array}$ & $\begin{array}{l}18.0 \\
(24.9)\end{array}$ & $\begin{array}{l}16.0 \\
(23.3)\end{array}$ & $\begin{array}{c}16.0 \\
(23.5)\end{array}$ & $\begin{array}{c}11.0 \\
(19.3)\end{array}$ & $\begin{array}{l}12.0 \\
(20.2)\end{array}$ \\
\hline $\begin{array}{l}\text { T6-Thiochloprid } \\
480 \text { SC }\end{array}$ & $200 \mathrm{ml}$ & $\begin{array}{l}6.7 \\
(2.6)\end{array}$ & $\begin{array}{l}5.7 \\
(2.4)\end{array}$ & $\begin{array}{l}5.6 \\
(2.4)\end{array}$ & $\begin{array}{c}7.0 \\
(2.8)\end{array}$ & $\begin{array}{c}4.0 \\
(10.8)\end{array}$ & $\begin{array}{c}3.2 \\
(9.9)\end{array}$ & $\begin{array}{c}5.0 \\
(12.7)\end{array}$ & $\begin{array}{c}3.5 \\
(10.4)\end{array}$ & $\begin{array}{c}3.8 \\
(10.9)\end{array}$ & $\begin{array}{c}28.0 \\
(31.9)\end{array}$ & $\begin{array}{c}27.0 \\
(31.3)\end{array}$ & $\begin{array}{c}28.0 \\
(31.9)\end{array}$ & $\begin{array}{c}26.0 \\
(29.9)\end{array}$ & $\begin{array}{c}20.0 \\
(26.4)\end{array}$ & $\begin{array}{l}18.0 \\
(24.9)\end{array}$ & $\begin{array}{c}18.0 \\
(25.1)\end{array}$ & $\begin{array}{c}12.0 \\
(20.2)\end{array}$ & $\begin{array}{l}12.0 \\
(20.2)\end{array}$ \\
\hline $\begin{array}{l}\text { T7-Triazophos } \\
\text { 25EC }\end{array}$ & 2.0 lit & $\begin{array}{l}7.0 \\
(2.6)\end{array}$ & $\begin{array}{c}5.9 \\
(2.4)\end{array}$ & $\begin{array}{l}5.8 \\
(2.4)\end{array}$ & $\begin{array}{c}7.2 \\
(2.8)\end{array}$ & $\begin{array}{c}4.0 \\
(10.8)\end{array}$ & $\begin{array}{c}3.8 \\
(10.9)\end{array}$ & $\begin{array}{c}5.0 \\
(12.7)\end{array}$ & $\begin{array}{c}4.0 \\
(11.2)\end{array}$ & $\begin{array}{c}4.5 \\
(12.0)\end{array}$ & $\begin{array}{c}27.0 \\
(31.3)\end{array}$ & $\begin{array}{c}26.0 \\
(30.6)\end{array}$ & $\begin{array}{c}27.0 \\
(31.7)\end{array}$ & $\begin{array}{l}28.0 \\
(31.8)\end{array}$ & $\begin{array}{c}22.0 \\
(27.8)\end{array}$ & $\begin{array}{c}22.0 \\
(27.8)\end{array}$ & $\begin{array}{c}16.0 \\
(23.5)\end{array}$ & $\begin{array}{l}15.0 \\
(22.7)\end{array}$ & $\begin{array}{c}16.0 \\
(23.5)\end{array}$ \\
\hline T8-Control & - & $\begin{array}{c}7.2 \\
(2.7)\end{array}$ & $\begin{array}{c}8.2 \\
(2.8)\end{array}$ & $\begin{array}{l}10.3 \\
(3 .)\end{array}$ & $\begin{array}{l}7.0 \\
(2.7)\end{array}$ & $\begin{array}{c}10.0 \\
(18.2)\end{array}$ & $\begin{array}{c}12.0 \\
(20.2)\end{array}$ & $\begin{array}{c}6.5 \\
(14.6)\end{array}$ & $\begin{array}{c}8.5 \\
(16.8)\end{array}$ & $\begin{array}{c}12.0 \\
(20.2)\end{array}$ & $\begin{array}{c}28.0 \\
(31.9)\end{array}$ & $\begin{array}{c}32.0 \\
(34.4)\end{array}$ & $\begin{array}{c}38.0 \\
(38.0)\end{array}$ & $\begin{array}{c}28.0 \\
(31.3)\end{array}$ & $\begin{array}{c}32.0 \\
(34.4)\end{array}$ & $\begin{array}{c}34.0 \\
(35.6)\end{array}$ & $\begin{array}{c}18.0 \\
(25.1)\end{array}$ & $\begin{array}{c}20.0 \\
(26.5)\end{array}$ & $\begin{array}{l}23.0 \\
(28.6)\end{array}$ \\
\hline C.D. & - & 0.11 & 0.09 & 0.12 & N/A & 1.28 & 1.27 & 0.31 & 1.84 & 1.11 & 2.99 & 2.37 & 2.51 & N/A & 1.73 & 4.38 & 1.39 & 0.39 & 0.52 \\
\hline $\mathrm{SE}(\mathrm{m})$ & - & - & - & & 0.08 & 0.43 & 0.43 & 0.10 & 0.62 & 0.37 & - & - & - & 1.86 & 0.57 & 1.46 & 0.46 & 0.13 & 0.17 \\
\hline $\operatorname{SE}(d)$ & - & 0.05 & 0.04 & 0.05 & 0.12 & 0.60 & 0.60 & 0.15 & 0.87 & 0.53 & 1.42 & 1.12 & 1.19 & 2.63 & 0.81 & 2.07 & 0.65 & 0.18 & 0.25 \\
\hline c.V & - & 2.62 & 2.35 & 3.15 & 5.14 & 6.40 & 6.60 & 1.35 & 9.84 & 5.25 & 6.29 & 5.36 & 5.3 & 10.81 & 3.95 & 9.73 & 3.32 & 1.11 & 1.44 \\
\hline
\end{tabular}

Values are mean of three replication of each treatment. Values in the paranthesis are square root transformed values for population and arcsine transformed values for percent damage. PTC- Pre treatment count, DAS-Days after spraying

Zadda et al., 2015; Nigude et al., 2018. Nataraja et al., (2013) found that thiamethoxam 25WG was effective in reducing thrips population in groundnut. Khanpara and his co-workers (2016) reported that spray of imidacloprid 200 SL @ 125 ml/ha or thiamethoxam 25 WG @ 200 gm ha $^{-1}$ or acephate 75 \% SP @ 500 gm ha $^{-1}$ at 15 days interval after initiation of pests were the most effective against thrips in groundnut. The effect of acephate $75 \%$ SP @ $1000 \mathrm{gm} \mathrm{ha}^{-1}$ on thrips population reduction was reported by Nigude et al., (2018).

Table 3. Natural enemies (Coccinellids; spider and its egg mass) population in newer molecules treated groundnut crop during rabi/summer 2012-15

\begin{tabular}{|c|c|c|c|c|c|c|c|c|c|c|c|c|c|}
\hline \multirow[b]{2}{*}{ Treatment } & \multirow[b]{2}{*}{ Doseha $^{-1}$} & \multicolumn{3}{|c|}{ Mean Coccinellids (no plant ${ }^{1}$ ) } & \multirow{2}{*}{ Mean } & \multicolumn{3}{|c|}{ Mean Spider (no plant ${ }^{-1}$ ) } & \multirow{2}{*}{ Mean } & \multicolumn{3}{|c|}{$\begin{array}{c}\begin{array}{c}\text { Mean Spider egg mass (no } \\
\text { plant }^{1} \text { ) }\end{array} \\
\end{array}$} & \multirow[b]{2}{*}{ Mean } \\
\hline & & $2012-13$ & 2013-14 & $2014-15$ & & $\begin{array}{c}2012- \\
13\end{array}$ & $\begin{array}{c}2013- \\
14\end{array}$ & $\begin{array}{c}2014- \\
15\end{array}$ & & 2012-13 & 2013-14 & 2014-15 & \\
\hline T1-Acephate $75 \% \mathrm{SP}$ & $1.30 \mathrm{~kg}$ & 0.66 & 0.20 & 0.03 & $\begin{array}{c}0.30 \\
(1.13)\end{array}$ & 0.30 & 0.03 & 0.05 & $\begin{array}{c}0.13 \\
(1.06)\end{array}$ & 0.25 & 0.02 & 0.02 & $\begin{array}{c}0.10 \\
(1.05)\end{array}$ \\
\hline T2-Acetamiprid $20 \%$ SP & $100 \mathrm{gm}$ & 0.46 & 0.27 & 0.04 & $\begin{array}{c}0.26 \\
(1.12)\end{array}$ & 0.50 & 0.03 & 0.03 & $\begin{array}{c}0.19 \\
(1.08)\end{array}$ & 0.21 & 0.03 & 0.03 & $\begin{array}{c}0.09 \\
(1.04)\end{array}$ \\
\hline T3-Fipronyl 5\% SC & $100 \mathrm{gm}$ & 0.61 & 0.33 & 0.03 & $\begin{array}{c}0.32 \\
(1.15)\end{array}$ & 0.36 & 0.03 & 0.05 & $\begin{array}{c}0.15 \\
(1.07)\end{array}$ & 0.21 & 0.01 & 0.04 & $\begin{array}{c}0.09 \\
(1.04)\end{array}$ \\
\hline T4-Imidacloprid 200SL & $200 \mathrm{ml}$ & 0.95 & 0.53 & 0.06 & $\begin{array}{r}0.51 \\
(1.22\end{array}$ & 0.68 & 0.07 & 0.07 & $\begin{array}{c}0.27 \\
(1.12)\end{array}$ & 0.25 & 0.02 & 0.04 & $\begin{array}{c}0.10 \\
(1.05)\end{array}$ \\
\hline T5-Thiamethoxam 25WG & $200 \mathrm{gm}$ & 0.86 & 0.27 & 0.06 & $\begin{array}{c}0.40 \\
(1.17)\end{array}$ & 0.66 & 0.03 & 0.03 & $\begin{array}{c}0.24 \\
(1.11)\end{array}$ & 0.32 & 0.01 & 0.04 & $\begin{array}{c}0.12 \\
(1.06)\end{array}$ \\
\hline T6-Thiochloprid 480SC & $200 \mathrm{ml}$ & 0.66 & 0.20 & 0.04 & $\begin{array}{c}0.30 \\
(1.13)\end{array}$ & 0.53 & 0.05 & 0.05 & $\begin{array}{c}0.21 \\
(1.09)\end{array}$ & 0.21 & 0.01 & 0.03 & $\begin{array}{c}0.08 \\
(1.04)\end{array}$ \\
\hline T7-Triazophos 25EC & 2.0 lit & 0.43 & 0.20 & 0.06 & $\begin{array}{c}0.23 \\
(1.11)\end{array}$ & 0.46 & 0.03 & 0.05 & $\begin{array}{c}0.18 \\
(1.08)\end{array}$ & 0.21 & 0.02 & 0.03 & $\begin{array}{c}0.09 \\
(1.04)\end{array}$ \\
\hline T8-Control & - & 1.16 & 0.80 & 0.11 & $\begin{array}{c}0.30 \\
(1.28)\end{array}$ & 0.73 & 0.07 & 0.09 & $\begin{array}{c}0.30 \\
(1.13)\end{array}$ & 0.523 & 0.04 & 0.05 & $\begin{array}{c}0.20 \\
(1.09)\end{array}$ \\
\hline & - & 0.02 & 0.02 & 0.01 & 0.08 & 0.020 & 0.00 & 0.05 & N/A & 0.018 & 0.00 & 0.09 & $\mathrm{~N} / \mathrm{A}$ \\
\hline C.D. & - & 0.01 & 0.01 & 0.00 & 0.03 & 0.007 & 0.00 & 0.02 & 0.017 & 0.006 & 0.00 & 0.03 & 0.013 \\
\hline $\mathrm{SE}(\mathrm{m})$ & - & 0.01 & 0.01 & 0.00 & 0.04 & 0.010 & 0.00 & 0.03 & 0.025 & 0.009 & 0.00 & 0.04 & 0.018 \\
\hline $\mathrm{SE}(\mathrm{d})$ & - & 0.89 & 1.21 & 0.33 & 4.12 & 0.962 & 0.00 & 2.31 & 2.768 & 0.958 & 0.00 & 4.45 & 2.138 \\
\hline
\end{tabular}

Values are mean of three replications of each treatment and mean of three time observation.

\section{Leafhopper}

Three years of data indicated that a significantly lesser population of leafhopper was recorded in all the treatments than in control after $7^{\text {th }}$ and $15^{\text {th }}$ DAS. However, the significantly low population of leafhopper 4.5, 3.2 and 2.0/ 3 terminal leaves/plant on $7^{\text {th }}$ DAS and $5.4,2.2$ and $2.5 / 3$ terminal leaves/ plant on $15^{\text {th }}$ DAS were recorded in imidacloprid 200SL @ $200 \mathrm{ml} \mathrm{ha}^{-1}$ treatment for all the three years, respectively. Next to imidacloprid, acetamiprid

$107|7-9| 316$ 
20SP@100 gm ha-1 recorded a low population of leafhopper 4.8, 3.2 and 2.5/ 3 terminal leaves/plant on $7^{\text {th }}$ DAS and 5.3,2.8 and 3.5/ 3 terminal leaves /plant on $7^{\text {th }}$ and $15^{\text {th }}$ DAS during rabi/summer 2012-13, 2013-14 and 2014-2015 respectively (Table 2). With reference to leafhopper damage percent imidacloprid recorded a minimum of $24 \%$ followed by acetamiprid (25\%) and acephate (25\%) during 2012-13. During the successive rabi/ summer season, acetamiprid 20\% SP, recorded $12 \%$ and $14.3 \%$, which was significantly different from the next treatment, imidacloprid $200 \mathrm{SL}$, which recorded $13.3 \%$ and $16.0 \%$ damage on $7^{\text {th }}$ and $15^{\text {th }}$ DAS. During 2014-15, imidacloprid and acetamiprid reduced the leafhopper damage to the tune of $8.0 \%$ to $8.5 \%$, which were significantly very low when compared to control (20-23\%). Thiamethoxam stood third in reducing the leafhopper incidence by about $16 \%$ and $12 \%$ during rabi/summer 2013-14 and 2014-15, respectively (Table 2).

Table 4. Effect of newer insecticide molecules on groundnut pod and haulm yield during rabi/summer seasons (2012-2015)

\begin{tabular}{|c|c|c|c|c|c|c|c|c|c|c|}
\hline \multirow[b]{2}{*}{ Treatment } & \multirow[b]{2}{*}{ Dose ha-1 } & \multicolumn{3}{|c|}{ Dry pod yield $\left(\mathrm{kg} \mathrm{ha}^{-1}\right)$} & \multirow{2}{*}{$\begin{array}{l}\text { Pooled } \\
\text { mean } \\
\left(\mathrm{kg} \mathrm{ha}^{-1}\right)\end{array}$} & \multicolumn{3}{|c|}{ Haulm yield $\left(\mathrm{t} \mathrm{ha}^{-1}\right)^{*}$} & \multirow{2}{*}{$\begin{array}{l}\text { Pooled } \\
\text { mean } \\
\left.(\mathrm{t} \mathrm{ha})^{-1}\right)\end{array}$} & \multirow[b]{2}{*}{ BCR } \\
\hline & & 2012-13 & 2013-14 & 2014-15 & & 2012-13 & 2013-14 & 2014-15 & & \\
\hline T1-Acephate 75\%SP & $1.30 \mathrm{~kg}$ & 2000 & 1808 & 1983 & 1930 & 8.8 & 7.4 & 6.8 & 7.6 & $1: 2.3$ \\
\hline T2-Acetamiprid 20\%SP & $100 \mathrm{gm}$ & 2216 & 2450 & 2158 & 2274 & 9.1 & 8.1 & 7.3 & 8.2 & $1: 2.8$ \\
\hline T3-Fipronyl 5\% SC & $100 \mathrm{gm}$ & 2133 & 1948 & 1983 & 2021 & 8.9 & 7.9 & 7.2 & 8.0 & $1: 2.5$ \\
\hline T4-Imidacloprid 200SL & $200 \mathrm{ml}$ & 2166 & 1890 & 1983 & 2013 & 8.8 & 7.6 & 6.6 & 7.6 & $1: 2.4$ \\
\hline T5-Thiamethoxam 25WG & $200 \mathrm{gm}$ & 2100 & 2217 & 1983 & 2100 & 8.4 & 7.7 & 7.3 & 7.8 & $1: 2.5$ \\
\hline T6-Thiochloprid 480SC & $200 \mathrm{ml}$ & 1916 & 2018 & 2158 & 2030 & 8.4 & 7.3 & 6.3 & 7.3 & $1: 2.4$ \\
\hline T7-Triazophos 25EC & 2.0 lit & 2133 & 1983 & 2158 & 2091 & 8.6 & 7.4 & 6.4 & 7.5 & $1: 2.5$ \\
\hline T8-Control & - & 1383 & 1692 & 1925 & 1666 & 7.5 & 7.2 & 7.0 & 7.2 & $1: 2.2$ \\
\hline C.D. & - & 200.4 & 218.7 & N/A & 246.3 & 126.2 & 463.5 & 189.3 & 461.1 & - \\
\hline $\mathrm{SE}(\mathrm{m})$ & - & 66.94 & 73.03 & 91.3 & 82.2 & 42.2 & 154.8 & 63.2 & 154.0 & - \\
\hline $\mathrm{SE}(\mathrm{d})$ & - & 94.7 & 103.3 & 129.1 & 116.3 & 59.6 & 218.9 & 89.4 & 217.8 & \\
\hline C.V. & - & 5.70 & 6.3 & 7.75 & 7.03 & 0.84 & 3.50 & 1.58 & 3.45 & - \\
\hline
\end{tabular}

Values are converted into tones from $\mathrm{kg} \mathrm{ha}^{-1}$

The present findings were in confirmation with the results of Saradava (2004), Venkanna et al. (2010) and Karena (2012), who reported that imidacloprid 0.005 per cent or thiamethoxam 0.05 per cent proved the most effective against leafhopper in groundnut. Nigude et al., (2018) indicated that imidacloprid $17.8 \mathrm{SL} @ 0.75 \mathrm{ml} / \mathrm{lit}$ was consistently most effective as compared to other treatments in reducing the survival population of leafhopper in groundnut. Similar positive effect of imidacloprid on leafhopper was reported in cotton. Imidacloprid and thiamethoxam proved significantly superior in controlling leafhopper in okra (Misra, 2002) and groundnut (Karuppuchamy, 2016).

\section{Natural enemies}

Pooled mean of the three years data indicated that significantly more no.of predatory coccinellids (0.51 plant $\left.^{-1}\right)$, spiders $\left(0.27\right.$ plant $\left.^{-1}\right)$, spider egg mass $\left(0.10\right.$ plant $\left.^{-1}\right)$ were recorded in imidacloprid 200SL followed by thiamethoxam 25WG, which recorded coccinellids 0.40 plant $^{-1}$, spider 0.24 plant ${ }^{-1}$ and spider egg mass (0.12 plant $^{-1}$ ) (Table $3)$. The reports of earlier research on the effect of newer molecules on coccinellids on different crops under field conditions were in conformity with the present study. Amirzade et al., (2014) reported that thiamethoxam toxicity to predatory coccinellids was lower than imidacloprid and acetamiprid. Munir ahmed et al., (2011) showed the least toxic effect of imidacloprid to the coccinellids. In contrast, Jadhav et al., (2018) reported that acetamiprid, thiamethoxam and imidacloprid were most toxic to the coccinellids in brinjal eco-system.

\section{Yield}

The pooled mean of pod and haulm yield for the three years data indicated that all the treatments gave the highest pod yield (>2000 kg $\left.\mathrm{ha}^{-1}\right)$ significantly. However, the maximum of pod yield (2216 kg ha-1) and haulm yield (8.1 $\mathrm{t} \mathrm{ha}^{-1}$ ) were recorded in acetamiprid 20\%SP followed by thiamethoxam (2100 kg ha-1 and $7.8 \mathrm{t} \mathrm{ha}^{-1}$ ) and imidacloprid 200SL (2013 kg ha-1 and $7.6 \mathrm{t} \mathrm{ha}^{-1}$ ) (Table 4). The highest BCR 1:2.8 was realized in acetamiprid $20 \%$ SP followed by thiamethoxam, triazophos (1:2.5) and imidacloprid, thiochloprid, novaluran (1:2.4) as against 1:2.2 in control. These findings were in accordance with the one made by Hanamant et al. (2014), which revealed that a reduction in the number of thrips caused enhanced pod and haulm yield of groundnut. Khanpara et al., (2016) also reported that thiamethoxam 25WG and acetamirprid 20SP were the economically viable 
treatment against thrips and jassids in groundnut.

\section{CONCLUSION}

Considering the effectiveness and economics of insecticides, spraying of imidacloprid 200SL @ 200 ml ha-1 or thiamethoxam 25 @WG 200 gm ha $^{-1}$ or acephate 75 \% SP @ $500 \mathrm{gm} \mathrm{ha}^{-1}$ or acetamiprid $20 \%$ SP @ $100 \mathrm{gm} \mathrm{ha}^{-1}$ at the time of initiation of pests were found to be the most effective against thrips and leafhoppers in groundnut. However, the natural enemies population was more in imidacloprid 200SL @ $200 \mathrm{ml} \mathrm{ha}^{-1}$ and thiamethoxam 25WG $200 \mathrm{gm} \mathrm{ha}{ }^{-1} \mathrm{gm} / \mathrm{ha}$. Thus, incorporation of newer chemistry molecules like imdiacloprid $200 \mathrm{SL}$ and thiamethoxam 25WG in integrated pest management programme for managing pests on groundnut may prove as economically viable with less interfering for the natural enemies in groundnut eco-system during rabi/summer seasons.

\section{REFERENCES}

Amirzade N, Izadi H, Jalali MA, H. Zohdi. 2014. Evaluation of three neonicotinoid insecticides against the common pistachio psylla, Agonoscena pistaciae, and its natural enemies. Journal of Insect Science 14: 35. http://www.insectscience. org/14.35

David B.V. and V. V. Ramamurthy. 2011. Elements of Economic Entomology. Namrutha publications, Chennai, Tamil Nadu. P: 385.

Hanamant, G., H Mahabaleshwar, H and R.A. Balikai. 2014. Yield loss and economic injury level (EIL) estimation for Thrips in groundnut crop. J. Exp. Zool. India 17(2): 683-686

Jadhav, R.D., Kadam, D.R., Jadhao P.B. and G.K. Kangale. 2018. Bioefficacy of newer insecticides against brinjal whitefly and their natural enemies. J. of Entomol. and Zool. Stud. 6(6): 1187-1191

Khanpara ,D.V. , Parmar, G.M., Chelani, M.K., and K.L. Dobariya.2016. Management of sucking pests in groundnut by foliar application with new insecticidal molecules. J. Farm Sci., 29(2): (280-282)
Karena, R.M., 2012. Seasonal incidence and bioefficacy of bio-pesticides alone and in combination with insecticides against major insect-pests of groundnut. Thesis Submitted to Junagadh Agricultural University, Junagadh, $81 \mathrm{pp}$.

Karuppuchamy, P. 2016. Ecologically Sustainable Pest Management for Thrips and Aphids in Groundnut. Madras Agric. J., 103 (4-6): 141-145

Misra H.P. 2002. Field evaluation of some newer insecticides against aphids (Aphis gossypii) and jassids (Amrasca biguttula) on okra. Indian J. Entomol. 64 (1): 80-84.

Munir Ahmad, Muhammad Rafiq, Arif M.I, A.H. Sayyed. 2011. Toxicity of some commonly used insecticides against Coccinella undecimpunctata (Coleoptera: Coccinellidae). Pakistan J. of Zoo. 43(6):11611165.

Nigude, V.K., S.P. Patil, S.A. Patil and A.S. Bagade. 2018. Management of Sucking Pest of Groundnut with Newer Molecules of Insecticides (Arachis hypogaea L.). Int.J.Curr.Microbiol.App.Sci. 7(01): 566-569. doi: https://doi.org/10.20546/ ijcmas.2018.701.068

Nataraj, M.V., Harish. G., Jasrotia. P., Holajjer. P., Savaliya. S.D. and M. Gajera. 2014. Neonicotinoids: A biorational approach for managing sucking insect-pests of groundnut. Ann. Pl. protec. Sci., 22(1): 42-48.

Sheoran, O.P; Tonk, D.S; Kaushik, L.S; Hasija, R.C and R.S. Pannu. 1998. Statistical Software Package for Agricultural Research Workers. Recent Advances in information theory, Statistics \& Computer Applications by D.S. Hooda \& R.C. Hasija Department of Mathematics Statistics, CCS HAU, Hisar (139-143)

Venkanna, Y., Ranga, G. V. and D. Reddy. 2010. Bioefficacy of neonicotinoid insecticides against thrips, Scirtothrips dorsalis and leafhoppers, Empoasca kerri in groundnut. Indian J. PI.Prot., 38: 68-72.

Zadda, K. and C. Vijayraghavan. 2015. Evaluation of newer insecticides against sucking insects infesting rainfed groundnut. Pestology 9: 45-48. 